\title{
Laparoscopic removal of an occult foreign body causing penetrating abdominal trauma in a child
}

\author{
Ram V. Anantha MD, Rodrick K. Lim MD, Neil H. Merritt MD
}

A n otherwise healthy four-year-old boy was brought to the emergency department by his mother several hours after he had an unwitnessed fall at home. He had been playing with his friends, when they found him bleeding through his shirt. He had no relevant past medical or surgical history, medication use or allergies. His vital signs were within normal limits, although he complained of abdominal pain.

On physical examination, his abdomen was soft, nondistended and diffusely tender, with no rebound tenderness or involuntary guarding. A small $1-\mathrm{cm}$ linear wound was found in the left upper quadrant of his abdomen, along the midclavicular line. A foreign body could not be felt despite careful examination, and penetration of the peritoneum could not be ruled out by local wound exploration. Faint bowel sounds were present, and he had an elevated leukocyte count (19.2 [normal 5-12] $\times 10^{9}$ cells/L); a differential count was not performed. His hemoglobin level was 130 (normal 110-160) g/L, his international normalized ratio was 1.1 (normal 0.9-1.1), and his serum aspartate aminotransferase was normal at 33 (normal $\leq 40$ ) U/L. His amylase (51 [normal 28-100] U/L) and lipase (17 [normal 1360]) levels were also within normal limits.

Because of the boy's abdominal pain and the emergency physician's suspicion of a possible penetrating injury, computed tomography (CT) of his abdomen and pelvis was performed, showing a $3 \times 1.5 \mathrm{~cm}^{2}$ foreign body in the left upper quadrant that appeared to penetrate the bowel (Figure 1). There was no free fluid or air, and there were no injuries to the spleen, liver, adrenal glands, pancreas or kidneys. The patient was transferred to a tertiary-care pediatric trauma centre for operative management. The plan was to remove the foreign body via laparoscopy, and, if a problem were found that could not be repaired by minimally invasive surgery, a full laparotomy would be performed.

After endotracheal intubation, the boy's abdominal cavity was examined by laparoscopy through a $12-\mathrm{mm}$ infraumbilical port. A thin shard of glass $\left(2 \times 6 \mathrm{~cm}^{2}\right)$ wrapped in omentum was visualized in the left upper quadrant (Figure 2). A second 5-mm port was placed through the wound site, and the foreign body was grasped and removed under direct visualization. Two additional $5-\mathrm{mm}$ ports were inserted in the left and right lower quadrants to facilitate mobilization and inspection of the small and large bowels. Thorough examination of the peritoneal cavity did not reveal any injury to the stomach, small intestine, retroperitoneum, colon, liver or spleen. The ports were removed, his abdomen was deflated, and the fascia and skin were closed. The patient resumed eating and drinking that evening. He was discharged home the following day, and he was able to tolerate a full diet, with no problems with bowel movements, and minimal pain. He was seen in clinic 1 month after his operation and was back to his normal activities.

\section{Discussion}

The care of children with abdominal injuries has evolved over the last 30 years, from mandatory open exploration in almost all cases to nonoperative and minimally invasive strategies. ${ }^{1}$ The morbidity and mortality rates $(20 \%$ and $5 \%$, respec-

\section{KEY POINTS}

- Computed tomography of the abdomen and pelvis should be considered for patients initially selected for nonoperative management of penetrating abdominal injuries to rule out intra-abdominal injuries and peritoneal violation.

- Laparoscopy can be helpful for identifying peritoneal violation in children with penetrating abdominal trauma, potentially precluding the need for laparotomy and, in the absence of any injury, leading to earlier mobilization, feeding and discharge.

- Laparoscopy can be a viable strategy for the removal of retained foreign bodies in select hemodynamically stable patients.

- In nonpenetrating or blunt abdominal trauma, laparoscopy can be used to identify and treat hollow viscus injuries in stable patients who have intra-abdominal fluid but no solid organ injury. 

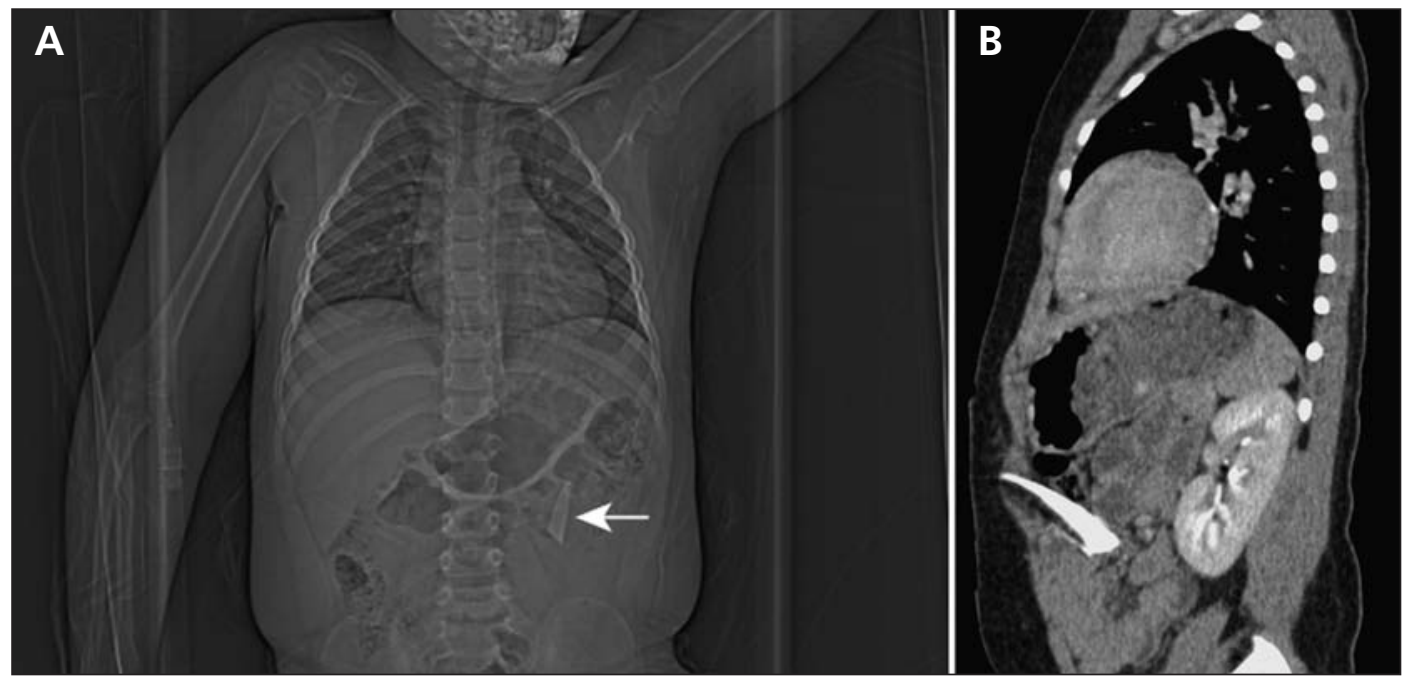

Figure 1: A computed tomogram of the abdomen and pelvis of a four-year-old boy showing a small, thin foreign object in the left upper quadrant (indicated by the white arrow) (A). The sagittal view (B) suggested a potential penetrating bowel injury by a foreign body, which was not detected by physical examination.

tively), ${ }^{2}$ the long-term risk of adhesive bowel obstruction $(3 \%)^{2}$ and the high incidence of nontherapeutic or negative laparotomies $(5 \%-53 \%)^{2}$ in children and adolescents reduced the use of laparotomy in trauma cases in this age group, and increased the use of options that did not involve open surgery. ${ }^{3}$ Imaging, especially CT imaging, has enhanced the diagnostic acumen of the initial evaluation. ${ }^{4}$ The use of modern laparoscopic techniques, first reported for diagnosing traumatic injuries in $1976^{5}$ and followed by many largescale studies from major trauma centres, ${ }^{6}$ has also contributed substantially to the minimally invasive management of abdominal trauma.

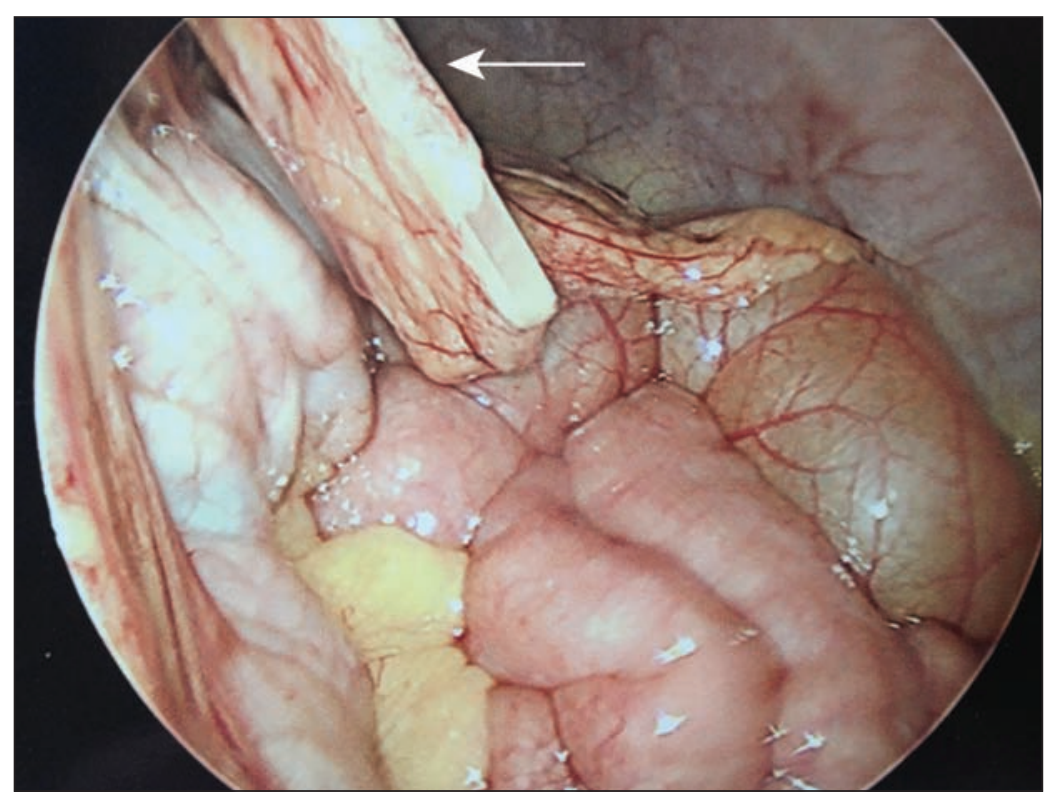

Figure 2: Laparoscopic examination of the abdominal cavity showed a long glass shard (white arrow; $2 \times 6 \mathrm{~cm}^{2}$ ) wrapped in omentum. No further injuries were identified, and the shard was successfully removed under direct visualization.

\section{Penetrating injuries}

Because there are no guidelines for the management of penetrating trauma in children, treatment algorithms for this population are typically derived from adult literature. ${ }^{1,7}$ For penetrating injuries, the indications for laparotomy are usually restricted to patients with peritonitis, free intra-abdominal air, organ injury with hemodynamic instability ${ }^{1}$ or retained foreign bodies such as knives. ${ }^{7}$ According to the Eastern Association for the Surgery of Trauma Practice Management Guidelines, hemodynamically stable patients (adults and children) with stab wounds or tangential gunshot wounds without signs of peritonitis or diffuse abdominal tenderness do not routinely warrant laparotomy (Figure 3 ). ${ }^{3}$

Computed tomography should be performed for patients selected for nonoperative care, because such scans can effectively screen for the presence of peritoneal penetration. ${ }^{3}$ Although the associated exposure to radiation warrants their careful use among children, ${ }^{8} \mathrm{CT}$ scans are superior to other types of imaging, including ultrasonography, for identifying penetrating trauma injuries. ${ }^{3}$

In our patient's case, the CT scan identified the occult glass shard, which had not been detected by physical examination, thus providing diagnostic clarity for a patient with suspected penetrating abdominal injury and pain. The young age of the patient and the absence of witnesses likely contributed to his inability to explain how he had been injured.

Studies involving adults and children have shown laparoscopy to be an effective way to diagnose and treat penetrating injuries to the diaphragm, solid organs and hollow viscera; cur- 
rent practice guidelines acknowledge its role in penetrating trauma (Figure 3). ${ }^{3}$ Our patient's case, in which an occult foreign body was removed by laparoscopy, shows the capabilities of a minimally invasive approach to treat injuries that would have typically required laparotomy. The glass shard was identified and successfully removed laparoscopically, avoiding full laparotomy and allowing the patient to be mobile and resume feeding earlier and to go home the day after surgery.

\section{Nonpenetrating injuries}

Laparoscopy is a sensitive and specific technique for evaluating and treating blunt or nonpenetrating abdominal injuries. ${ }^{1}$ Such injuries can be chal- lenging to diagnose and manage in children because the injuries may be confounded by equivocal findings on physical examination, associated neurologic or extra-abdominal injuries, and the presence of progressing injuries such as hollow viscus or mesenteric injuries. ${ }^{4,9}$

Computed tomography is the optimal test for evaluating solid organs within the abdomen and retroperitoneum, but it is not as reliable for identifying bowel injuries. ${ }^{4,8}$ Diagnostic peritoneal lavage, which involves the insertion of a catheter into the peritoneal cavity, can accurately identify intra-abdominal hemorrhage or bowel injury, but it is invasive and has a high rate of false-positive results. ${ }^{10}$ Focused abdominal sonography for trauma (FAST) can be per-

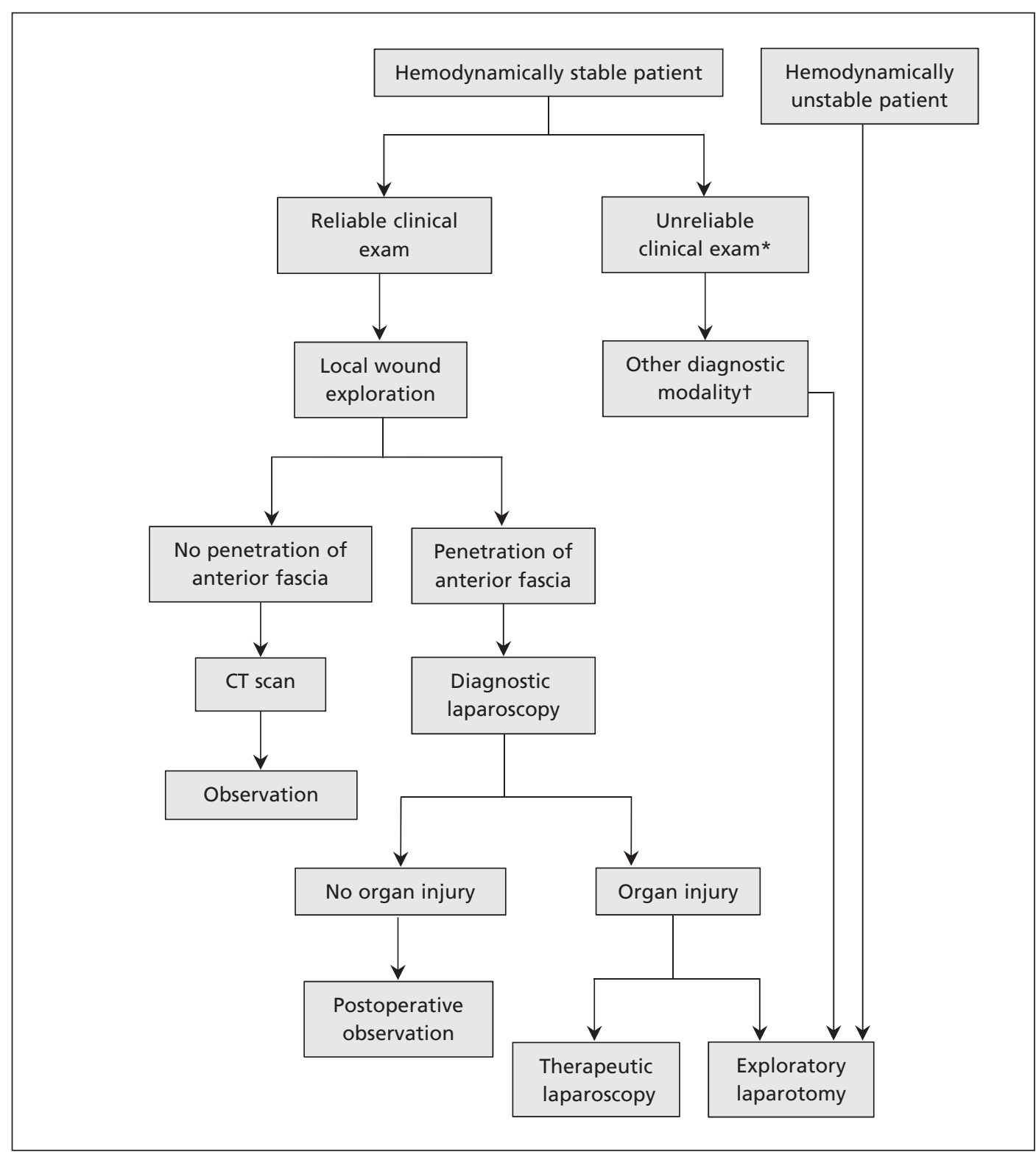

Figure 3: An algorithm for the management of penetrating abdominal trauma in children (adapted from Feliz and colleagues, ${ }^{1}$ with premission from Elsevier). ${ }^{3}$ *Patients with, for example, brain or spinal cord injury, intoxication or need for sedation. tIncludes computed tomography or diagnostic peritoneal lavage. 
formed noninvasively and quickly, but it is unreliable for identifying organ-specific injury and cannot replace CT scans. ${ }^{1,10}$

Although laparotomy is the treatment of choice for hemodynamically unstable patients with suspected intra-abdominal injuries, laparoscopy may be an effective treatment strategy for stable patients with intra-abdominal fluid but no solid organ injury detected by $\mathrm{CT}^{1}{ }^{1}$ In such patients, laparoscopy can identify and aid in treating hollow viscus injuries, while avoiding nontherapeutic laparotomies. ${ }^{1,5}$

\section{References}

1. Feliz A, Shultz B, McKenna C, et al. Diagnostic and therapeutic laparoscopy in pediatric abdominal trauma. J Pediatr Surg 2006; 41:72-7.

2. Renz BM, Feliciano DV. Unnecessary laparotomies for trauma: a prospective study of morbidity. J Trauma 1995;38:350-6.

3. Como JJ, Bokhari F, Chiu WC, et al. Practice management guidelines for selective nonoperative management of penetrating abdominal trauma. J Trauma 2010;68:721-33.

4. Taylor GA, Fallat ME, Potter BM, et al. The role of computed tomography in blunt abdominal trauma in children. $J$ Trauma 1988;28:1660-4.

5. Gazzaniga AB, Stanton WW, Bartlett RH. Laparoscopy in the diagnosis of blunt and penetrating injuries to the abdomen. Am J Surg 1976;131:315-8.

6. Leppäniemi A, Haapiainen R. Diagnostic laparoscopy in abdominal stab wounds: a prospective, randomized study. J Trauma 2003;55:636-45.

7. Clarke SC, Stearns AT, Payne C, et al. The impact of published recommendations on the management of penetrating abdominal injury. Br J Surg 2008;95:515-21.

8. Livingston $\mathrm{MH}$, Igric A, Vogt $\mathrm{K}$, et al. Radiation from CT scans in paediatric trauma patients: indications, effective dose, and impact on surgical decisions. Injury 2013 Jul 8. pii: S0020-1383 (13)00288-X

9. Ross SE, Dragon GM, O'Malley KF, et al. Morbidity of negative coeliotomy in trauma. Injury 1995;26:393-4.

10. Hoff WS, Holevar M, Nagy KK, et al. Practice management guidelines for the evaluation of blunt abdominal trauma: the East practice management guidelines work group. J Trauma 2002;53:602-15.

Affiliations: Division of General Surgery (Anantha), Division of Pediatric Surgery (Merritt), London Health Sciences Centre; Department of Pediatrics and Medicine (Lim), Children's Hospital, London Health Sciences Centre, London, Ont.

Contributors: Ram Anantha wrote the manuscript. Rodrick Lim and Neil Merritt participated in the care of the patient upon transfer to the tertiary care trauma centre. All authors contributed to the drafting and revising of the manuscript and approved the final version submitted for publication.

The section Cases presents brief case reports that convey clear, practical lessons. Preference is given to common presentations of important rare conditions, and important unusual presentations of common problems. Articles start with a case presentation (500 words maximum), and a discussion of the underlying condition follows (1000 words maximum). Visual elements (e.g., tables of the differential diagnosis, clinical features or diagnostic approach) are encouraged. Written consent from patients for publication of their story is a necessity and should accompany submissions. See information for authors at www.cmaj.ca.

\section{How you can get involved in the CMA!}

The CMA is committed to providing leadership for physicians and promoting the highest standard of health and health care for Canadians. To strengthen the association and be truly representative of all Canadian physicians the CMA needs to hear from members interested in serving in elected positions and on appointed committees and advisory groups. The CMA structure comprises both governing bodies and advisory bodies either elected by General Council or appointed by the CMA Board of Directors. The Board of Directors - elected by General Council — has provincial/territorial, resident and student representation, is responsible for the overall operation of the CMA and reports to General Council on issues of governance.

CMA committees advise the Board of Directors and make recommendations on specific issues of concern to physicians and the public. Five core committees mainly consist of regional, resident and student representation while other statutory and special committees and task forces consist of individuals with interest and expertise in subject-specific fields. Positions on one or more of these committees may become available in the coming year.

For further information on how you can get involved please go to http://www.cma.ca/membercentre/how-you-can-get-involved, or contact

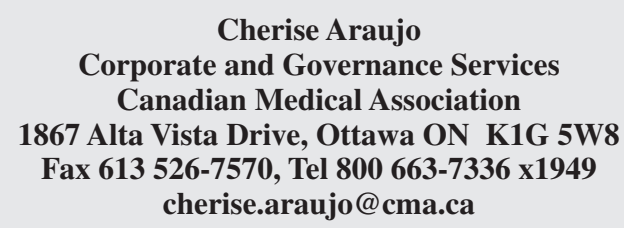

By getting involved, you will have an opportunity to make a difference.

ASSOCIATION
MÉDICALE
CANADIENNE

We hope to hear from you! 